\title{
Independent control of acceleration and direction of the hand when hitting moving targets
}

\author{
ELI BRENNER*, MARC H. E. DE LUSSANET and JEROEN B. J. SMEETS \\ Vakgroep Fysiologie, Erasmus Universiteit Rotterdam, Postbus 1738, \\ NL-3000 DR Rotterdam, The Netherlands
}

Received 20 February 2001; revised 11 May 2001; accepted 28 May 2001

\begin{abstract}
Human subjects were asked to hit moving targets as quickly as they could. Nevertheless the speed with which the subjects moved toward identical stimuli differed between trials. We examined whether the subjects compensated for a lower initial acceleration by aiming further ahead of the target. We found that the initial acceleration of the hand and its initial direction were hardly correlated. Thus subjects did not aim further ahead when they hit more slowly. This supports our earlier suggestion that the acceleration of the hand and the direction in which it moves are controlled separately.
\end{abstract}

Keywords: Motor control; interception; timing; arm movement; human.

\section{INTRODUCTION}

Intercepting moving targets is special in that it requires co-ordination between time and place. If subjects are to intercept a target at a certain place, they must make sure to arrive at that place, as well as to be there at the right time (Peper et al., 1994; Tresilian, 1994; Carnahan and McFadyen, 1996; Port et al., 1997). If there are no such restrictions, a suitable combination of time and place will be based on postural and task constraints. For instance, in order to hit a target as hard as possible in a given direction, subjects must attempt to reach the target when their own velocity is maximal (Lee et al., 1983; Michaels et al., 2001). This involves a high degree of co-ordination between various aspects of the task (Bernstein, 1967), which could be expected to result in correlations. For instance, when trying to hit a moving target, the selected time and place are inseparable; by selecting a suitable time one also selects a place, and vice versa. Thus the two must be correlated.

\footnotetext{
*To whom correspondence should be addressed. E-mail: brenner@ fys.fgg.eur.nl
} 
We previously found that in fast interception the acceleration of the hand and the direction in which it moves are based on different visual information. The acceleration is based on the target's speed whereas the direction is based on its position. This became particularly evident when the target's apparent velocity, but not its apparent position at each instant, was manipulated by moving the background. This influenced the acceleration of the hand, but not its initial direction of movement (Smeets and Brenner, 1995). This and similar findings suggest that the acceleration of the hand is controlled independently of the direction in which it moves (Bairstow, 1987; Brenner and Smeets, 1996). Similar arguments have been raised for an independent control of the direction and extent of reaching movements (Favilla et al., 1990; Favilla and De Cecco, 1996; Messier and Kalaska, 1997).

The simplicity of separating the control of the hand into several independent mechanisms, each based on a particular kind of visual information, is attractive. It simplifies the link between the visual information and the controlled aspect of the movement, so that the neural processing can presumably be done faster and more reliably. Short visuo-motor delays make it feasible to adjust ongoing movements on the basis of new visual information that becomes available as the movement progresses (such as unpredictable changes in a target's motion or visual information about the hand's position relative to the target).

In the present study, we examine a prediction of a complete separation into independent mechanisms. This prediction is that the variability in the initial acceleration of subjects' movements and the variability in the initial direction of the movements should not be correlated. We examine whether this is so by analysing the variability in repeated hits toward identical targets. If the two mechanisms are not independent we expect subjects to direct their hand further ahead of the target on trials in which they move more slowly, because on such trials the target will have moved further by the time the hand reaches the surface on which they are moving.

\section{METHODS}

\section{Procedure}

Twelve subjects each took part in 3 sessions. In each session they hit 120 moving targets (virtual spiders) with a rod $(22 \mathrm{~cm}$ long; $0.9 \mathrm{~cm}$ radius). They were explicitly instructed to hit the targets as quickly as possible. The target always moved to the right at $15 \mathrm{~cm} / \mathrm{s}$ across a visible background. In each session 20 targets appeared at each of 6 possible initial positions, in random order. Subjects started with their hand not further than $5 \mathrm{~cm}$ from a prescribed position, about $40 \mathrm{~cm}$ from the screen across which the targets moved (for technical details of target presentation see Brenner et $a l ., 1998$ ). The positions at which the target could appear (and all other target positions) were defined relative to where the subject actually initially held the rod on that trial. Thus the required direction of movement did not depend on where the subject initially held the rod. The rod's initial distance from the screen did vary modestly between trials. 


\section{Feedback}

Subjects could see their hand's contour occluding part of the image when the hand was close to the screen. If the centre of the rod hit the screen within $1.8 \mathrm{~cm}$ of the centre of the target, the spider appeared to have been 'squashed'. If subjects hit to the left of the spider it ran away to the right. If they hit above the spider it ran away downwards. And so on.

\section{Measuring hand movements}

The position of the rod's tip was determined at $250 \mathrm{~Hz}$ from the measured positions of two active infrared markers (resolution better than $0.1 \mathrm{~mm}$ ) with an Optotrak 3010 (Northern Digital Inc.) movement analysis system. Subjects held the rod between their fingers and thumb as they would hold a pen. Fifty-two of the 4320 trials were discarded for various reasons (primarily markers disappearing from view during the movement or the subject starting to move before the target appeared).

\section{The key variables}

For each trial we first determined the moment the rod started to move (velocity threshold of $0.2 \mathrm{~m} / \mathrm{s}$ in the direction of the screen), and the target's position and the rod's distance from the screen at that moment. Next, we determined the average acceleration of the rod and the direction in which it moved during the first $48 \mathrm{~ms}$ (12 samples) of the movement (hereafter referred to as the rod's initial acceleration and movement direction). Finally, we determined how long it took the rod to reach the screen from the moment it started moving (the movement time) and where the rod hit the screen relative to where the target was at that moment (position of hit).

\section{Dealing with variability in reaction time}

If the hand's acceleration and direction of movement are controlled in a co-ordinated manner, we expect to find a negative correlation between the variations in the rod's initial acceleration and the variations in how far ahead of the target that the rod is heading. The former variation can be determined directly from the measured initial accelerations, but for the latter it is not enough to know the direction in which the rod started to move. The complicating factor is that the target will also be at different positions on different trials (due to variability in the reaction time). To be able to account for this we have to express the target's position at the moment that the rod started to move and the direction in which the rod started to move in the same units. Thus we need to know how many centimetres further to the right (on the screen) that the rod is heading when it starts to move a measured angle further to the right. This is not simple trigonometry, because the rod's path is curved. However. we can estimate this relationship, because we expect it to be systematic (with most of the lateral displacement taking place during the beginning of the movement; Smeets 


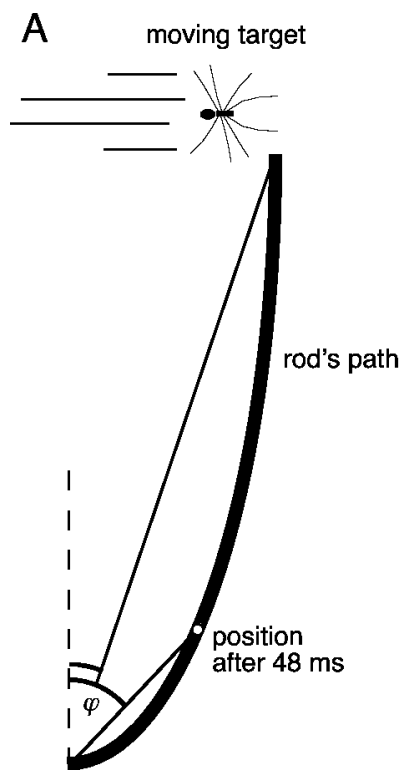

B

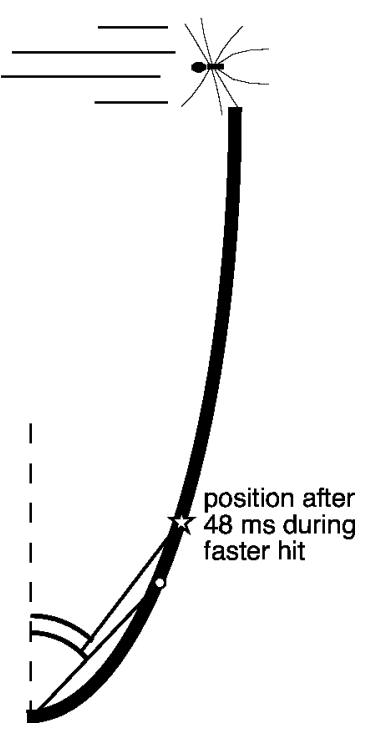

Figure 1. Schematic representation of the hand's path. Left: illustration of why the initial direction $(\varphi)$ exaggerates the eccentricity of the target. Right: illustration of how a faster hit could appear to be heading less far ahead of the target ( smaller angle $\varphi$ ) due to the curvature of the path.

and Brenner, 1995). An exaggerated example of the kind of path we expect for the rod is shown schematically in Fig. 1A.

We used the six different initial positions of the targets to determine how much further to the right the rod was heading when it started to move a measured angle further to the right. For each subject, session and initial target position we determined the average initial direction of the rod's movement $(\bar{\varphi})$ and the target's average position at the moment that the rod starts to move $(\bar{x})$. For each subject and session this gave us 6 pairs of values, one for each initial target position. Fitting a line to the relation between these values gave us an estimate of how much further along the target's path the rod was heading when it moved a given angle further to the right $(\mathrm{d} \bar{\varphi} / \mathrm{d} \bar{x})$. For each trial this was then combined with the rod's initial movement direction $(\varphi)$ and the target's position at the moment that the rod started to move $(x)$ to give an estimate of how much further ahead of the target the rod was heading $(\Delta h)$.

$$
\Delta h=\frac{(\varphi-\bar{\varphi})}{\mathrm{d} \bar{\varphi} / \mathrm{d} \bar{x}}-(x-\bar{x})
$$

\section{The correlation}

Equation (1) gives an estimate of how far ahead of the target the rod was initially heading on a given trial, relative to the average distance ahead of the target for that subject, session and initial target position. For the initial acceleration we calculated 
a comparable measure by subtracting the mean acceleration for that subject, session and initial target position from the value on the given trial. Expressing both as deviations from the mean for that subject, session and initial target position means that we can calculate a single correlation for all the data without having to worry about correlated differences between subjects, sessions or initial target positions. This correlation reflects the relationship between variations in the rod's initial acceleration and variations in its initial movement direction for hits by individual subjects toward targets with identical initial positions within one session.

\section{Dealing with a possible influence of path curvature on the correlation}

The method described above removes almost all possible sources of spurious correlation. However, the curved paths themselves could introduce some correlation (see Fig. 1B). If subjects move faster on a given trial, the rod will be further along the path after $48 \mathrm{~ms}$. For movements to the right of straight-ahead (as shown), the angle $\varphi$ will be smaller on faster trials, which will incorrectly be interpreted as hitting less far ahead of the target. For movements to the left of straight-ahead a smaller angle will incorrectly be interpreted as hitting further ahead of the target. This could introduce a net correlation if the two do not cancel each other, and will certainly introduce additional variability.

The change in angle with the position along the path, and thus with the initial acceleration, decreases as the path becomes less curved. In order to reduce the impact of the above-mentioned correlations we therefore conducted an additional analysis in which we selected trials on which the rod initially moved within $2.5 \mathrm{deg}$ of a straight path towards the screen (the deviations were now calculated with respect to the means from within the selection). Such a selection has the additional advantage that the accelerations that are being considered are along very similar paths, so that there is very unlikely to be any systematic influence of arm biomechanics. A trial was discarded if it was the only one that was selected for that subject, session and initial target position, because in that case there was no deviation from the mean that could be used in the correlation.

\section{RESULTS}

The symbols in Fig. 2 show the average lateral target position (relative to the initial lateral position of the rod) at the moment that the rod started to move, and the average initial direction of the rod's movement, for each initial target position. This is the average of all subjects and sessions. The thick line shows a linear fit to these points. The thin line indicates the direction in which the rod would move if it moved straight towards the target's average position at the moment that the rod started to move. The dashed line indicates the direction in which the rod would move if it moved straight towards the average position at which the target was hit. The slope of the latter two lines is much shallower than that of the fit to the actual initial 


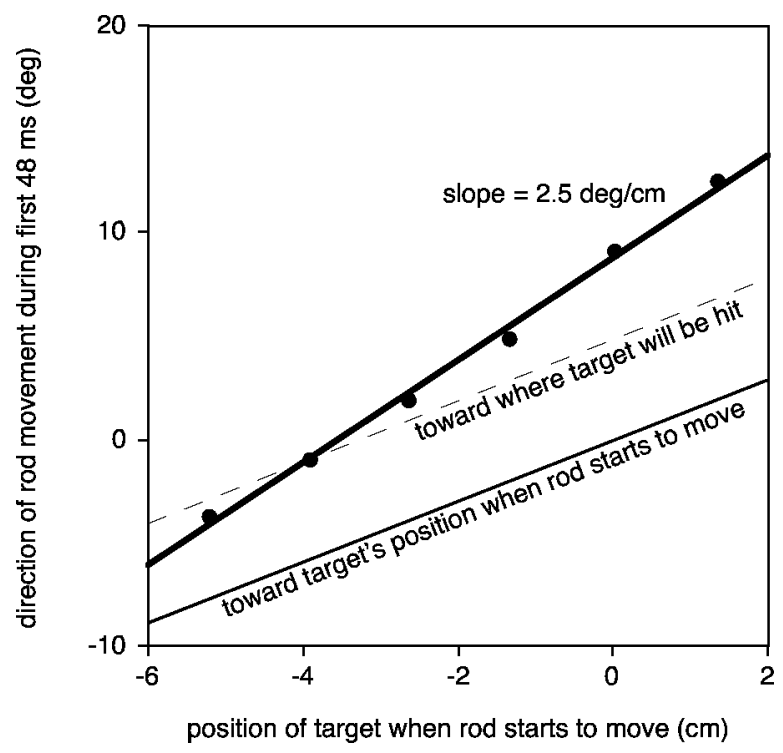

Figure 2. The initial direction in which the rod moved as a function of the target's position when it started to move. Symbols: average data for each of the six initial target positions. Thick line: linear fit to the average data. Thin line: the direction in which the rod would have moved if it had moved straight toward the target's position at the moment that the rod started to move. Dashed line: the direction in which it would have moved if it had moved straight toward the target's position when the rod hit the screen.

directions, indicating that the paths were indeed curved in the manner that is shown schematically in Fig. 1 ( $\varphi$ is about 3 times as large after $48 \mathrm{~ms}$ than it would be if the hand had moved straight toward the target). The intersection between the thick and dashed lines is approximately at $0 \mathrm{deg}$, which is defined as the direction perpendicular to the screen. This indicates that on average subjects started their movement straight toward the screen when they hit the target straight in front of the rod.

Figure 3 shows the deviation from the rod's mean initial acceleration and the deviation from the mean distance ahead of the target that it is heading $(\Delta h)$ for all 4268 trials. The value of $\mathrm{d} \bar{\varphi} / \mathrm{d} \bar{x}$ that was used to determine $\Delta h$ (see equation (1)) is the slope of a fit such as that shown by the thick line in Fig. 2, but this was done separately for the data of each subject and session. There was a small negative correlation between the deviation from the rod's mean initial acceleration and $\Delta h$, but it was certainly not striking. Moreover, the rod initially moved to the right on most trials (see values in Fig. 2), which itself could lead to a negative correlation (see methods and Fig. 1B).

To circumvent the latter issue we analysed the selection of 505 trials on which the initial direction of the rod's movement was not more than $2.5 \mathrm{deg}$ of straight-ahead. All subjects were represented within this selection, though one subject with only 2 trials (the others contributed between 24 and 70 trials). Figure 4 shows the deviation 


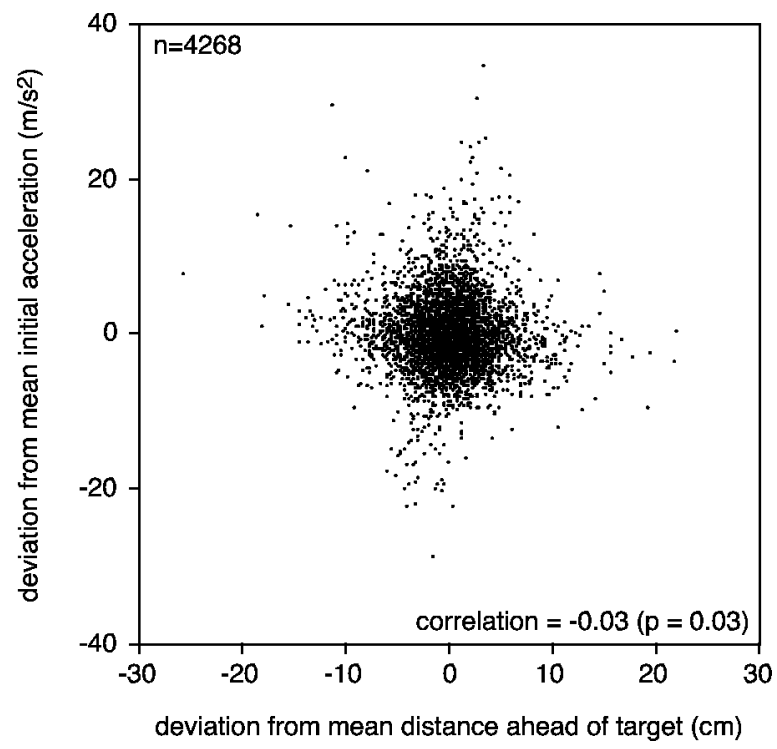

Figure 3. The correlation between the rod's acceleration and its initial movement direction for all trials. Each symbol shows the deviation from the mean initial acceleration of the rod, and the deviation from the mean distance ahead of the target that it was aiming $(\Delta h)$, on a single trial. The deviations are calculated with respect to the mean value for that subject during that session for that initial target position.

from the mean initial acceleration and the deviation from the mean distance ahead of the target $(\Delta h)$ for these trials. The correlation is larger than in Fig. 3, but it is still hardly striking. However, this correlation cannot be due to the rod predominantly moving to the right.

In order to get a better impression of what the modest correlation means, we estimated the relationship that is to be expected if subjects adjust their acceleration to their path, or vice versa. To do so we determined the extent to which the acceleration would have to change to compensate for moving the rod farther or less far ahead of the target. If the rod is moved a distance $\Delta h$ farther or less far ahead of the target than average, and $v$ is the target's velocity, the movement time has to change from the average value $\overline{M T}$ to a new value $M T$, where

$$
M T-\overline{M T}=\frac{\Delta h}{v} .
$$

If we assume a constant acceleration (Brenner et al., 1998), we can directly relate this change in movement time to a prediction for the change in acceleration $(\Delta a)$.

$$
\Delta a=\frac{2 D}{M T^{2}}-\frac{2 \bar{D}}{\overline{M T}^{2}} .
$$

In equation (3), $D$ and $\bar{D}$ are the rod's initial distance from the screen on that trial and on average, respectively. ${ }^{1}$ By substituting $M T$ in equation (3) by its value 


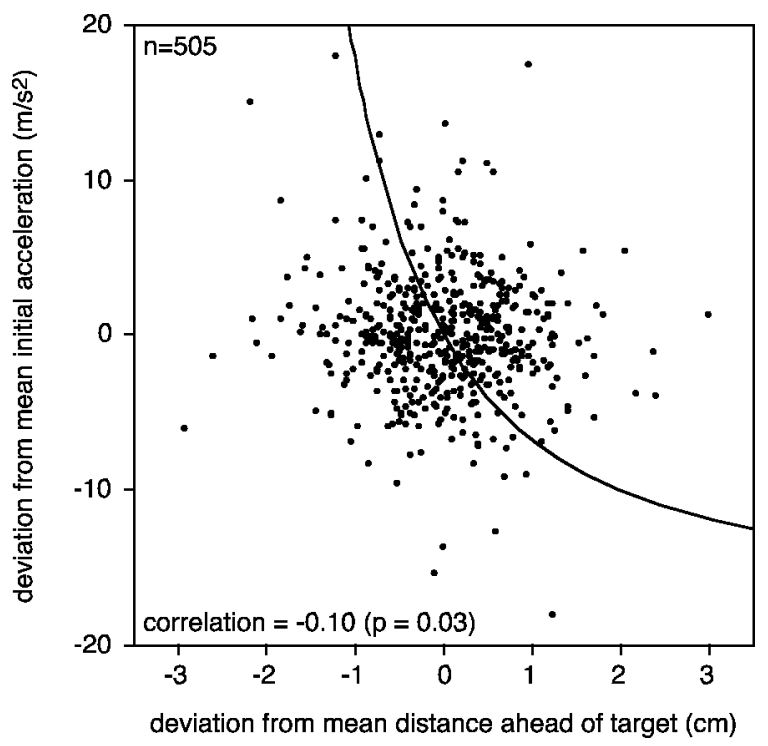

Figure 4. The correlation between the rod's acceleration and its initial movement direction for the trials that started within $2.5 \mathrm{deg}$ of straight-ahead. For further details see legend of Fig. 3 . The curve gives an impression of the expected relationship.

obtained from equation (2) we get

$$
\Delta a=\frac{2 D}{\left(\overline{M T}+\frac{\Delta h}{v}\right)^{2}}-\frac{2 \bar{D}}{\overline{M T}^{2}} .
$$

The curve in Fig. 4 shows this relationship for the overall mean movement time and initial distance of the hand, ignoring all variability in both measures. The curve does not appear to bear any relationship with the data points. Using the overall mean values neglects differences between subjects, sessions and initial target positions for $\overline{M T}, D$ and $\bar{D}$, as well as differences between individual trials (for $D$ ). Ignoring the variability in $D$ and $\bar{D}$ makes very little difference. The standard deviation in the distance from the screen is $1.8 \mathrm{~cm}$, at an average distance of $38.5 \mathrm{~cm}$. The mean movement time is $218 \mathrm{~ms}$. Assuming a constant acceleration of the rod with no adjustment for its initial distance, the variability in the initial distance from the screen will give rise to a standard deviation in the movement time of about $5 \mathrm{~ms}$, which corresponds with a variability in target position of only $0.075 \mathrm{~cm}$.

Ignoring the variations in $\overline{M T}$ does make a difference. Equation (4) is quite sensitive to the value of $\overline{M T}$, which differs considerably across subjects. As a final step we therefore calculated the required change in acceleration $\Delta a$ that was necessary to compensate for the value of $\Delta h$ and $D$ on each trial (using $\overline{M T}$ and $\bar{D}$ for that subject, session and initial target position). We determined the correlation between this predicted value and the measured deviation from the mean initial acceleration. They were not correlated (correlation $=-0.07 ; p=0.12$ ). Thus 
the variations in the initial acceleration of the rod between trials do not compensate for the variations in how far ahead of the target that the rod is heading.

\section{DISCUSSION}

Although the correlation between initial direction and acceleration was not striking, it was statistically significant. Since the correlation increased when we selected the straightest paths, it is unlikely to have anything to do with the paths' curvature. The lack of quantitative correspondence between the changes in initial direction and acceleration (as evident from the last analysis) implies that it is also unlikely to be due to co-ordinated changes of initial acceleration and direction. A possible origin for the correlation is a change in strategy. Our analysis is based on the assumption that our subjects' strategy does not change during the experiment, so that the correlation arises from co-ordination within single trials. We tried to ensure this by emphasising to our subjects that they had to hit as fast as possible. However, this instruction is ambiguous, because the subject does have to hit the target. If a subject misses several spiders, or if he gets tired, he is likely to intentionally decrease his initial acceleration. Even if he does not immediately change his initial direction as well, he will presumably do so after several trials. This will give rise to a negative correlation in our analysis. Note that this source of correlation does not refute an independent control of acceleration and direction.

Thus we interpret our results as supporting the idea that the acceleration of the hand and the direction in which it moves are controlled independently (Bairstow, 1987; Smeets and Brenner, 1995; Brenner and Smeets, 1996). Of course there are limitations on how independently they can be controlled if the subject is required to fulfil a task. However, at least in the case of hitting moving targets, the variability apparently does not arise at the level at which the two aspects are co-ordinated to meet the task requirements.

\section{Implications with regard to variability in movement time}

We here show that subjects do not adjust the direction in which they move their hand to variations in acceleration, and thus in movement time. The variability in movement time, even when individual subjects hit under identical conditions, may therefore limit their performance. Our subjects' mean movement times varied from about 150 to about $280 \mathrm{~ms}$. The standard deviation in the movement time increased with the movement time from about $10 \mathrm{~ms}$ to about $40 \mathrm{~ms}$. Since we have just shown that this variability in movement time is independent of the direction in which the hand moves, we expect it to give rise to variability in the position of the hit (relative to the target). An estimate of this variability is shown by the solid symbols in Fig. 5 $\left(S D_{t}\right.$; calculated by multiplying the standard deviation of the movement time by the target velocity; each symbol represents one subject). The actual measured standard deviations are shown by the open symbols $\left(S D_{\mathrm{m}}\right)$. Assuming that the measured 


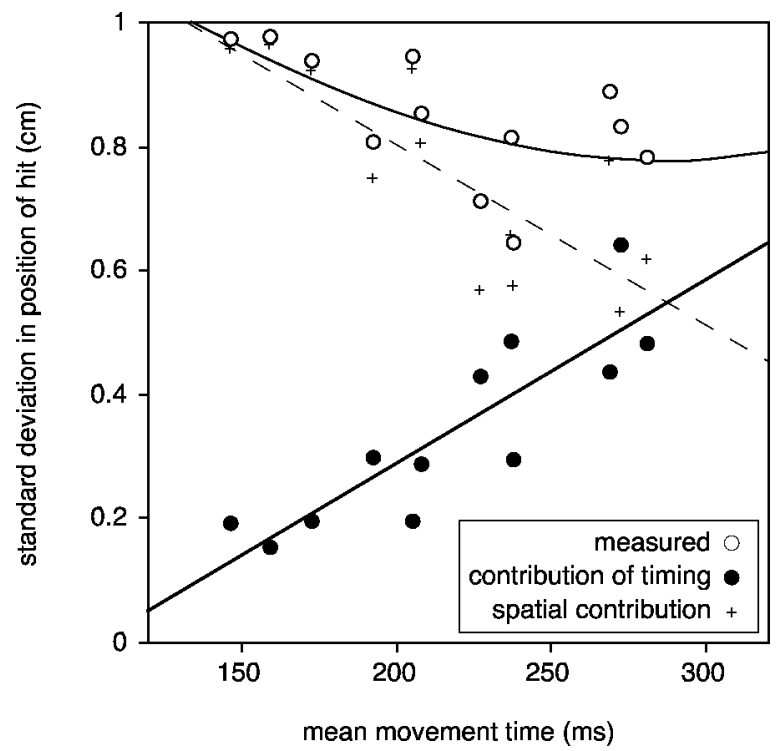

Figure 5. The standard deviation in where the rod hit the screen relative to the target (lateral component only) as a function of the subject's average movement time. Each symbol shows the value for one subject. Open symbols: measured values. Solid symbols: proposed contribution of variability in movement time. Crosses: proposed contribution of spatial variability. For details see text.

standard deviation is the result of a combination of the above-mentioned temporally based variability and an independent spatial variability $\left(S D_{\mathrm{s}}\right)$,

$$
S D_{\mathrm{m}}^{2}=S D_{\mathrm{t}}^{2}+S D_{\mathrm{s}}^{2},
$$

we can estimate the magnitude of the spatial variability (crosses in Fig. 5) from the other two.

The thick solid straight line in Fig. 5 is a linear fit for the relationship between mean movement time and the temporally based variability in hitting position (for support for a linear fit see Schmidt et al., 1979). The dashed line is a similar fit for the spatial variability (Schmidt et al., 1979). The thin solid curve shows the relationship between mean movement time and the combined variability as calculated from these two fits using equation (5). From this curve we can determine that the optimal movement time in terms of spatial error is $286 \mathrm{~ms}$. Most subjects had lower movement times, presumably because they abided by the instruction to hit as quickly as possible despite the potential increase in spatial errors.

The spatial error that results from a given timing error increases linearly with the target's velocity. The optimal movement time therefore also depends on the target's velocity. On the basis of the preceding analysis, the optimal movement time can be calculated to be about $12 \mathrm{~ms}$ shorter (or longer) for every $1 \mathrm{~cm} / \mathrm{s}$ increase (or decrease) in target velocity. This could explain why subjects tend to move faster toward fast targets than toward slow ones (Smeets and Brenner, 1995; Brenner et al., 1998). The dashed line and the curve in Fig. 5 converge at the shortest 
movement times, indicating that the contribution of variability in timing to the total spatial variability is negligible for short movement times. This explains why the tendency to move faster toward fast targets is not evident for subjects who hit very fast (Brouwer et al., 2000).

Subjects have often been asked to hit moving targets as quickly as possible (e.g. van Donkelaar et al., 1992; Smeets and Brenner, 1995). This instruction forces subjects to seek a trade-off between moving fast (as instructed) and hitting the target (for which there is an optimal movement time). The linear fits in Fig. 5 are calculated across subjects, assuming that the movement time influences each kind of variability in the same way for all subjects. The reasonable fits support this assumption. Differences between subjects can therefore be attributed to differences in how they deal with the trade-off between speed and accuracy.

The above analysis of the variability in movement time may explain why the control of the hand's acceleration and direction are not co-ordinated. Figure 5 shows that the variability in movement time is not a very important source of spatial variability in our task (especially for subjects who move fast). The benefit of adjusting the direction of movement to the variability in timing would therefore probably be quite limited.

\section{Acknowledgement}

This research was supported by grant 575-23-002 of the Foundation for Behavioural and Educational Sciences of The Netherlands Organisation for Scientific Research (NWO).

\section{NOTE}

1. Since the acceleration is assumed to be constant, we now estimate it from the movement time. The correlation between this estimate and the measured initial acceleration was 0.85 .

\section{REFERENCES}

Bairstow, P. J. (1987). Analysis of hand movement to moving targets, Human Movement Science 6, 205-231.

Bernstein, N. (1967) The Co-ordination and Regulation of Movements. Pergamon Press, Oxford.

Brenner, E. and Smeets, J. B. J. (1996). Hitting moving targets: co-operative control of 'when' and 'where', Human Movement Science 15, 39-53.

Brenner, E., Smeets, J. B. J. and de Lussanet, M. H. E. (1998). Hitting moving targets: continuous control of the acceleration of the hand on the basis of the target's velocity, Experimental Brain Research 122, 467-474.

Brouwer, A., Brenner, E. and Smeets, J. B. J. (2000). Hitting moving objects: The dependency of hand velocity on the speed of the target, Experimental Brain Research 133, 242-248.

Carnahan, H. and McFadyen, B. J. (1996). Visuomotor control when reaching toward and grasping moving targets, Acta Psychologica 92, 17-32. 
van Donkelaar, P., Lee, R. G. and Gellman, R. S. (1992). Control strategies in directing the hand to moving targets, Experimental Brain Research 91, 151-161.

Favilla, M. and De Cocco, E. (1996). Parallel direction and extent specification of planar reaching arm movements in humans, Neuropsychologia 34, 609-613.

Favilla, M., Gordon, J., Hening, W. and Ghez, C. (1990). Trajectory control in targeted force impulses. VII. Independent setting of amplitude and direction in response preparation, Experimental Brain Research 79, 530-538.

Lee, D. N., Young, D. S., Reddish, P. E., Lough, S. and Clayton, T. M. H. (1983). Visual timing in hitting an accelerating ball, Quart. J. Exper. Psychol. 35A, 333-346.

Messier, J. and Kalaska, J. F. (1997). Differential effect of task conditions on errors of direction and extent of reaching movements, Experimental Brain Research 115, 469-478.

Michaels, C. F., Zeinstra, E. B. and Oudejans, R. R. D. (2001). Information and action in punching a falling ball, Quart. J. Exper. Psychol. 54A, 69-93.

Peper, L., Bootsma, R. J., Mestre, D. R. and Bakker, F. C. (1994). Catching balls: how to get the hand to the right place at the right time, J. Exper. Psychol.: Human Perception and Performance 20, $591-612$.

Port, N. L., Lee, D., Dassonville, P. and Georgopoulos, A. P. (1997). Manual interception of moving targets. I. Performance and movement initiation, Experimental Brain Research 116, 406-420.

Schmidt, R. A., Zelaznik, H. and Hawkins, B. (1979). Motor-output variability: A theory for the accuracy of rapid motor acts, Psychol. Rev. 86, 415-451.

Smeets, J. B. J. and Brenner, E. (1995). Perception and action based on the same visual information: distinction between position and velocity, J. Exper. Psychol.: Human Perception and Performance 21, 19-31.

Tresilian, J. R. (1994). Approximate information sources and perceptual variables in interceptive timing, J. Exper. Psychol.: Human Perception and Performance 20, 154-173. 